hep-ph/0307022

MSU-HEP-030101

October 10, 2018

BNL-NT-03/2

RBRC-325

\title{
CTEQ6 Parton Distributions with Heavy Quark Mass Effects
}

\author{
Stefan Kretzer ${ }^{a, b, c}$, H.L. Lai ${ }^{d}$, Fredrick I. Olness ${ }^{e}$, W.K. Tung ${ }^{a}$ \\ ${ }^{a}$ Department of Physics and Astronomy, Michigan State University, \\ East Lansing, MI 48824 USA \\ ${ }^{b}$ Physics Department, Brookhaven National Laboratory, \\ Upton, New York 11973, USA \\ ${ }^{c}$ RIKEN-BNL Research Center, Bldg. 510a, Brookhaven National Laboratory, \\ Upton, New York 11973 - 5000, USA \\ ${ }^{d}$ Taipei Municipal Teacher's College, Taipei, Taiwan \\ ${ }^{e}$ Department of Physics, Southern Methodist University, Dallas, Texas, 75275 USA
}

Previously published CTEQ6 parton distributions adopt the conventional zero-mass parton scheme; these sets are most appropriate for use with massless hard-scattering matrix elements commonly found in most physics applications. For precision observables which are sensitive to charm and bottom quark mass effects, we provide in this paper an additional CTEQ6HQ parton distribution set determined in the more general variable flavor number scheme which incorporates heavy flavor mass effects. The results are obtained by combining these parton distributions with consistently matched DIS structure functions computed in the same scheme. We describe the analysis procedure, examine the predominant features of the new distributions, and compare with previous distributions. 
Contents

\begin{tabular}{|l|l|}
\hline I. Introduction & 3 \\
\hline
\end{tabular}

II. The Generalized $\overline{M S}$ Scheme with non-zero mass heavy flavor partons

A. Leading contributions and the $\operatorname{ACOT}(\gamma)$ implementation 4

$\begin{array}{ll}\text { B. Other contributions to the full NLO calculation } & 6\end{array}$

$\begin{array}{ll}\text { C. Remaining scheme and scale choices } & 6\end{array}$

III. Global Fitting and the CTEQ6HQ Parton Distributions 7

\begin{tabular}{ll|}
\hline IV. Comparison with data & 8
\end{tabular}

A. DIS Charm Production Data from HERA 9

B. Neutrino Data in Global Parton Analvses $\quad 9$

V. Comparison with related PDFS: CTEQ6HQ, CTEQ6M, CTEQ5HQ 11

$\begin{array}{ll}\text { A. Light partons at the input scale } & 11\end{array}$

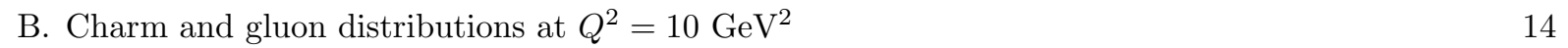

C. $Q$-dependence of heavv quark mass effects 14

\begin{tabular}{|l|l|}
\hline VI. Concluding Remarks & 16
\end{tabular}

$\begin{array}{ll}\text { Acknowledgment } & 16\end{array}$

$\begin{array}{ll}\text { References } & 16\end{array}$ 


\section{INTRODUCTION}

Parton distributions provide the essential link between the theoretically calculated partonic crosssections, and the experimentally measured physical cross-sections involving hadrons and mesons. This link is crucial if we are to make incisive tests of the standard model, and search for subtle deviations which might signal new physics. Since perturbative calculations are renormalization scheme dependent, ${ }^{1}$ it is important to use properly matched hard-scattering cross-sections and parton distribution functions (PDFs) in evaluating factorized cross-sections for physical applications. This issue is particularly relevant for applications involving heavy quarks, since the heavy quark introduces a new mass scale which leads to complications of the PQCD formalism.

For most physical applications, it is convenient (and a good approximation) to use hard-scattering cross-sections calculated in the zero parton-mass limit. In fact, next-to-leading-order hard-scattering crosssections in the non-zero quark-mass case have only been calculated for some very basic processes, such as deep inelastic scattering (DIS); they are not yet available for most interesting "new physics" applications. Thus, the most useful parton distributions for general applications are those determined in a global QCD analysis using the zero quark-mass approximation. This was the choice made for the latest series of CTEQ6 parton distributions: CTEQ6M, CTEQ6D, and CTEQ6L. 1] For definiteness, this scheme will be referred to as the zero-mass variable-flavor-number scheme (ZM-VFNS).

This paper extends Ref. [1] by performing a similar global analysis using the generalized (non-zero quark-mass) $\overline{M S}$ perturbative QCD framework of Refs. [2, 3, 4, 5, 6], which we label the general-mass variable-flavor-number scheme (GM-VFNS). When matched to the corresponding hard-scattering crosssections calculated in the same scheme, the combination should provide a more accurate description of the precision DIS structure function data, as well as other processes which are sensitive to charm and bottom mass effects. The main result we present here is a new set of parton distributions evaluated in this GM-VFNS which we identify as CTEQ6HQ. ${ }^{2}$

Perturbative QCD with non-zero quark-masses is well-established in the literature. 2, 4, 5, 9, 10, 11, 12. Its implementation is, however, necessarily more complicated than the corresponding zero-mass case. In addition to the familiar renormalization-scheme dependence, more ambiguities arise from the implementation of the charm and bottom thresholds (in relation to their masses), and the detailed way the 3-flavor, 4-flavor, and 5 -flavor renormalization schemes are matched at the transition scales. Several schemes proposed in the recent literature are formally equivalent to each other at high energies; but, they can differ in the threshold region, as well as in the intermediate energy range, where much of the experimental data lie. [4, 6, 11, 12, 13, 14] Some of these schemes are more natural and numerically robust, e.g. insensitive to variations of the remaining parameters (such as scale), than others. We follow the procedure proposed in Refs. 14, 15. which has been shown to be particularly stable as it naturally takes into account the dominant (logarithmic and non-logarithmic) mass-dependent kinematic effects with a physically motivated choice of the scaling variable.

Section 2 summarizes the method of analysis. Because this work represents an extension of the zero quark-mass CTEQ6 global QCD analysis, we shall focus mostly on the new elements related to non-zero quark mass; details that are in common with the massless case can be found in Ref. [1]. Section 3 describes the global analysis of data. Section 4 compares the new parton distributions with data. Section 5 makes comparisons between the CTEQ5M, CTEQ6M and CTEQ6HQ PDFs. At asymptotically high energies, mass effects become negligible; therefore, all generalized parton model prescriptions should approach the

\footnotetext{
${ }^{1}$ We shall use the term renormalization scheme in the general sense to include both the usual renormalization (ultra-violet subtraction) scheme, and the factorization (infrared and collinear subtraction) scheme.

2 A fit named CTEQ6F3 employing the three fixed-flavor-number scheme (3-FFNS) for DIS (cf., Refs. 7, 8]) which is suitable for special applications in that scheme, will be presented separately.
} 
same zero quark-mass limit. We show that the differences between CTEQ6M and CTEQ6HQ PDFs indeed vanish with increasing $Q$, as expected. This section also contains a discussion of applications and issues related to uncertainties of physics predictions resulting from these parton distributions.

\section{THE GENERALIZED $\overline{M S}$ SCHEME WITH NON-ZERO MASS HEAVY FLAVOR PARTONS}

The main feature of this analysis, compared to the standard zero-mass parton CTEQ6 PDF sets, is the adoption of the generalized perturbative QCD framework incorporating non-zero mass effects for the charm and bottom quarks. ${ }^{3}$ Collins has shown that, to all orders of the perturbation expansion, factorization of the DIS structure functions holds for massive partons to the same degree of rigor as in the familiar zeromass case. 5| The relatively simple physical ideas underlying the full factorization proof are described in Refs. 6, 14, 15]. To implement this formalism, which we shall refer to as the generalized $\overline{M S}$ scheme (or GM-VFNS, as mentioned earlier), we follow the explicit procedures outlined in the Appendix of Ref. [6], and supplement this with the threshold prescription of Refs. 14, 15]. The latter reference also contains a review of alternate approaches.

The NLO DIS structure functions are given by the generic formula (suppressing the structure function label $1,2,3)$,

$$
F(x, Q)=\sum_{a} \int \frac{d z}{z} f_{a}(z, \mu) \hat{\omega}^{a}\left(\frac{x}{z}, \frac{Q}{\mu}, \frac{m_{H}}{\mu}, \alpha_{s}(\mu)\right)+\mathcal{O}\left(\alpha_{s}^{2}, \frac{\Lambda^{2}}{Q^{2}}, \frac{\Lambda^{2}}{m_{H}^{2}}\right)
$$

where " $a$ " is the initial state parton label, $f_{a}$ is the parton distribution function, $\hat{\omega}^{a}$ is the hard-scattering cross-section calculated in PQCD to order $\alpha_{s}^{1}, \Lambda$ is the QCD-lambda parameter, and $m_{H}$ (generically) represents heavy quark masses, if present. The prescription-dependence allowed by the PQCD formalism is associated with possible implementations of the first term on the right-hand side of Eq. 1 within the accuracy specified by the remainder term (which will be dropped from now on). ${ }^{4}$ In the sum over partons in Eq. 1] contributions due to the light quark $(u, d, s)$ are standard; contributions arising from massive charm and bottom quarks, along with that of the gluon, are treated differently, as explained below.

\section{A. Leading contributions and the $\operatorname{ACOT}(\chi)$ implementation}

To be specific, we will consider DIS neutral-current charm production process. The leading-order process is a virtual-photon scattering off a charm parton: $\gamma^{*} c \rightarrow c$. The NLO QCD corrections for this process are the boson-gluon fusion process $\left(\gamma^{*} g \rightarrow c \bar{c}\right)$, and the gluon radiation process $\left(\gamma^{*} c \rightarrow c g\right)$ as well as the charm-initated one-loop virtual process $\left(\gamma^{*} c \rightarrow c\right)$.

First, let us examine the kinematic region near the charm production threshold: $W=\sqrt{Q^{2}(1 / x-1)} \simeq$ $2 m_{c}$. To make the notation precise, we will use the renormalization scale $\mu$ to generically label our characteristic energy scale; separately, we use $\mu_{m}$ to label the matching point, and $\mu_{t}$ to label the transition point (cf. [6] and below). To implement the generalized $\overline{M S}$ scheme, the first step is to consider a 3-flavor scheme (appropriate for energy scales $\mu \lesssim m_{c}$ ), a 4-flavor scheme (for scales $\mu \gtrsim m_{c}$ ), and choose a matching point $\mu_{m}$ (on the order of $m_{c}$ ) where the two schemes are matched (i.e., where the discontinuities of $\alpha_{s}(\mu)$ and $f_{a}(x, \mu)$ are calculated). We choose the conventional value for the matching point, $\mu_{m}=m_{c}$, which yields the simplest matching conditions. [2]

\footnotetext{
3 The top quark can, for all practical purposes, be treated as a heavy particle, not a parton.

${ }^{4}$ Note, according to the factorization proof of Ref. [5], the remainder term contains corrections of order $\Lambda^{2} / Q^{2}$ and $\Lambda^{2} / m_{H}^{2}$, but no corrections of order $m_{H}^{2} / Q^{2}$.
} 
Having precisely defined the 3-flavor and 4-flavor schemes, which co-exist in the region of the charm threshold, we can still choose a transition point, $\mu_{t}$, where one makes the transition from the 3 -flavor to the 4 -flavor scheme in the calculation of physical quantities. ${ }^{5}$ The choice of the transition point is arbitrary-its choice is part of the definition of the composite renormalization scheme. While there is much flexibility in the choice of $\mu_{t}$, clearly it must lie within the overlapping region of applicability of the 3 -flavor and 4-flavor schemes - therefore, we typically choose $\mu_{t} \sim m_{c}$. Although a case can be made, in principle, for choosing $\mu_{t}$ far above $m_{c}$ (since the 4-flavor scheme is not a natural scheme just above $m_{c}$ ), or for considering an $x$ dependent transition scale, $\mu_{t}\left(x, m_{c}\right)$ (because the threshold $W>2 m_{c}$ depends on $x$ as well as $Q$ ), we make the plain choice $\mu_{t}=m_{c}$ to simplify the calculation. This choice is reasonable only within a prescription for handling $x$-dependent threshold effects of Eq. 10 which naturally suppresses the 4-flavor contribution to the physical structure function when $\mu \sim m_{c}$ and/or $W \gtrsim 2 m_{c}$. The specific renormalization prescription of Ref. 14] has precisely this feature, as we shall outline below.

Detailed descriptions of the various NLO contributions to the the generalized $\overline{M S}$ scheme were discussed in Ref. [6]. While we refrain from reproducing them here, we do need to specify the precise way the threshold effects are implemented when heavy quarks are involved. Consider first the numerically significant NLO boson-gluon fusion contribution $\left(\gamma^{*} g \rightarrow c \bar{c}\right)$ :

$$
\begin{aligned}
F^{(1)}\left(\gamma^{*} g \rightarrow c \bar{c}\right) & =\int \frac{d z}{z} g(z, \mu) \hat{\omega}_{g}\left(\frac{x}{z}, \frac{Q}{\mu}, \frac{m_{c}}{\mu}\right) \\
& =\alpha_{s}(\mu)\left[\int_{\chi}^{1} \frac{d z}{z} g(z, \mu) \omega_{g}^{1}\left(\frac{x}{z}, \frac{m_{c}}{Q}\right)-\ln \left(\frac{\mu}{m_{c}}\right) \int_{\zeta}^{1} \frac{d z}{z} g(z, \mu) P_{g \rightarrow c}\left(\frac{\zeta}{z}\right) \omega_{c}^{0}\left(\frac{m_{c}}{Q}\right)\right]
\end{aligned}
$$

where the first term on the right-hand side corresponds to the unsubtracted boson-gluon-fusion diagram contribution (with the $\alpha_{s}$ factor explicitly taken out), ${ }^{6}$ and the second term represents the subtraction term which renders $\hat{\omega}_{g}$ infra-red safe. The variable $\chi$ is dictated by the kinematics of the boson-gluon-fusion partonic subprocess $\left(\gamma^{*} g \rightarrow c \bar{c}\right)$ to be a generalized scaling (or "rescaling") variable

$$
\chi=x\left(1+\frac{4 m_{c}^{2}}{Q^{2}}\right)
$$

where $x$ is the conventional Bjorken variable. In contrast, the other scaling variable, $\zeta$, in Eq. 2 can, in principle, be chosen arbitrarily provided the following two constraints are imposed.

1. $\zeta \rightarrow x$ in the high energy limit.

2. The same $\zeta$ variable is used here as in the order $\alpha_{s}^{0}$ ("leading-order") process $\gamma^{*} c \rightarrow c$.

In the high energy limit $\left(m_{c}^{2} / Q^{2} \rightarrow 0\right)$, the first constraint ensures that both $\zeta \rightarrow x$ and $\chi \rightarrow x$ such that the complete boson-gluon fusion contribution is infra-red safe. The leading-order process $\gamma^{*} c \rightarrow c$ is:

$$
F^{(0)}\left(\gamma^{*} c \rightarrow c\right)=\int \frac{d z}{z} c(z, \mu) \hat{\omega}_{c}^{0}\left(\frac{Q}{\mu}, \frac{m_{c}}{\mu}\right) \delta\left(1-\frac{\zeta}{z}\right)=c(\zeta, \mu) \hat{\omega}_{c}^{0}\left(\frac{Q}{\mu}, \frac{m_{c}}{\mu}\right) .
$$

For the second constraint, if the same $\zeta$ variable is used for both the boson-gluon fusion process of Eq. (2) $\left(\gamma^{*} g \rightarrow c \bar{c}\right)$ and the leading-order $\left(\gamma^{*} c \rightarrow c\right)$ contribution of Eq. (4), then the gluon subtraction term

\footnotetext{
5 The distinction between the matching and the transition points is not addressed in most papers. This distinction is made in Ref. [5]; it is discussed at length in the Appendix of Ref. [6]. As mentioned above, arguably, there is a good case for choosing the transition point $\mu_{t}$ to be higher than the simple matching point $\mu_{m}=m_{c}$.

${ }^{6}$ We use the notation that, for each flavor, $\omega_{a}$ denotes the parton-level cross-section before infrared and collinear subtraction, while $\hat{\omega}_{a}$ denotes the corresponding infra-red safe cross-section after subtraction.
} 
(last term in Eq. 2) cancels the leading-order contribution in the limit $\mu \rightarrow m_{c}$; this ensures that the 4flavor formula will reduce to the 3-flavor formula, and the dominant contribution in this region will be the unsubtracted gluon fusion term, as required by the correct physics to this order of PQCD.

A natural choice of $\zeta$ that ensures the best behavior at both the high and the low energy limits is proposed by Ref. [14]:

$$
\zeta=\chi \equiv x\left(1+4 m_{c}^{2} / Q^{2}\right)
$$

This choice has the property that as the heavy quark production threshold is approached from above ( $W \rightarrow$ $2 m_{c}$ ), then $\zeta \rightarrow 1$ such that the integration measure in Eq. 2 vanishes. ${ }^{7}$ Therefore, with the choice $\zeta=\chi$, the subtraction term in Eq. 2 and the LO quark term, Eq. 4] individually vanish at threshold by kinematics. In addition, the cancellation between these two terms due to the dynamics of PQCD, as explained in Ref. [4], still operates.

This results in unequaled stability of the predictions in the threshold region, compared to previous implementations of the GM-VNFS in the threshold region [4, 12, 13, 16]. These usually give rise to rather prescription-sensitive behavior just above threshold because the perturbation-expansion-inspired prescriptions generally overlook heavy quark production kinematic requirements, while the physically important gluon fusion term (in the threshold region) is strongly kinematically-suppressed. The robust implementation we adopt will be referred to as the "ACOT $(\chi)$ " scheme, following Refs. [14, 15]. ${ }^{8}$

\section{B. Other contributions to the full NLO calculation}

For the complete order $\alpha_{s}$ calculation, we must also include the real and virtual NLO quark-initiated contribution to Eq. [1]

$$
F^{(1)}\left(\gamma^{*} c \rightarrow c+X\right)=\int \frac{d z}{z} c(z, \mu) \hat{\omega}_{c}^{1}\left(\frac{x}{z}, \frac{Q}{\mu}, \frac{m_{c}}{\mu}\right)
$$

The hard-scattering cross-section, $\hat{\omega}_{c}^{1}\left(x, \frac{Q}{\mu}, \frac{m_{c}}{\mu}\right)$, has been computed for general masses 17] in the ACOT scheme [4]. For scales around the canonical DIS choice of $\mu \simeq Q$, this term is numerically insignificant compared to the terms considered above. We include it in our calculation to ensure (perturbative) consistency.

It is also worth mentioning that the calculation of this term, which is computation-intensive in spite of the smallness of its numerical contribution, can be considerably streamlined by using a simplifying feature of PQCD in the generalized $\overline{M S}$ scheme: one can set $m_{c}=0$ in $\hat{\omega}_{c}^{1}$ and $\omega_{c}^{0}$ in Eq. (2) and Eq. (4) without sacrificing accuracy. This point is discussed in detail in Ref. [18] where the numerical insensitivity of the calculation to the choice of $m_{c}$ is explicitly demonstrated. ${ }^{9}$

\section{Remaining scheme and scale choices}

The only remaining arbitrariness of our prescription is now associated with the familiar choice of the factorization (and renormalization) scale $\mu$. The scale dependence of perturbative calculations in general arise from imperfect matching between the terms in the truncated perturbation series. Because of the near-perfect matching in the $\operatorname{ACOT}(\chi)$ prescription discussed above (in contrast to alternate choices), the

\footnotetext{
7 This is more easily seen from the equivalent formula $\chi=1-(1-x)\left(1-4 m_{c}^{2} / W^{2}\right)$.

8 So named since it is a variant of the original ACOT approach [4], replacing the naive $x$ with the kinematically natural rescaling variable $\chi$ of Eq. 3

9 The simplified scheme [18] can also be recovered by investigation of the resummed perturbation series. 15.
} 
dependence of the physical structure functions on the choice of $\mu$ is completely negligible (cf., Ref. 14]) throughout the energy range of our global analysis. For definiteness, we use $\mu^{2}=Q^{2}+m_{c}^{2}$. In summary, the quark initiated terms in the simplified $\operatorname{ACOT}(\chi)$ scheme adopted in this work are:

$$
\left.\int_{\chi}^{1} \frac{d z}{z} c(z, \mu) \hat{\omega}_{c}^{0,1}\left(\frac{\chi}{z}, \frac{Q}{\mu}, \frac{m_{c}}{\mu}=0\right)\right|_{\mu^{2}=Q^{2}+m_{c}^{2}}=\left.\int_{\chi}^{1} \frac{d z}{z} c(z, \mu) C_{\overline{\mathrm{MS}}}^{0,1}\left(\frac{\chi}{z}, \frac{Q}{\mu}\right)\right|_{\mu^{2}=Q^{2}+m_{c}^{2}}
$$

with $C_{\overline{\mathrm{MS}}}^{0,1}$ representing the standard coefficient functions for massless quarks in LO and NLO, defined in the conventional $\overline{M S}$ scheme.

The same treatment is applied to the $b$-quark threshold region. Here, the 4 -flavor scheme is matched onto the 5-flavor scheme for scales larger than $m_{b}$, and the production of open bottom particles in the final state is added to the inclusive DIS structure functions. In principle, a similar treatment needs to be applied for other processes included in the global analysis (currently, Drell-Yan and jet production) using appropriate hard-scattering cross-sections calculated in the massive scheme. In practice, this is not done for two reasons: (i) NLO calculations of the hard-scattering cross-section involving massive quarks do not yet exist for these processes; and (ii) unlike the case of DIS, the experimental errors in these processes are relatively large compared to the anticipated differences between the massless and massive scheme calculations.

\section{GLOBAL FITTING AND THE CTEQ6HQ PARTON DISTRIBUTIONS}

The new global fitting performed in the generalized $\overline{M S}$ formalism follows the same procedure as that of the earlier CTEQ6 analysis [1] using the zero-mass parton formalism. The data sets used before are supplemented by: (i) the H1 $F_{2}^{e^{+} p}$ set [19] (which was inadvertently left out in Ref. [1]); and (ii) the H1 20] and ZEUS 21] data sets for the structure function $F_{2}^{c}$ with tagged charm particles in the final state. The additional $\mathrm{H} 1 F_{2}^{e^{+} p}$ data set does not have much influence on the new analysis, since one already obtains an excellent fit to these data just by comparing them with predictions of CTEQ6M. The $F_{2}^{c}$ data sets are quite relevant for this analysis since $F_{2}^{c}$ is sensitive to the charm and gluon distributions, which are tightly coupled in the generalized $\overline{M S}$ formalism. ${ }^{10}$

The parametrization of the non-perturbative parton distribution functions at $Q_{0}=m_{c}=1.3 \mathrm{GeV}$ is the same as in Ref. 1]. For this study, we assume as usual that charm partons are entirely "radiatively generated" (i.e., through QCD evolution) from the starting scale $Q_{0}$ onward. This assumption is somewhat arbitrary, and it is obviously dependent on the choice of $Q_{0}$. The possibility for having a small component of non-perturbative charm [22] at low $Q$, and its physical consequence will be examined separately.

As in the previous CTEQ6 analysis, correlated experimental systematic errors are fully incorporated whenever available. The best fit obtained with these inputs shall be called CTEQ6HQ, or C6HQ for short. A broad measure of the quality of this fit is provided by the overall $\chi^{2}$ of 2033 for a total number of 1950 data points $\left(\chi^{2} / \mathrm{DOF}=1.04\right)$. This is to be compared to a $\chi^{2}$ of 1946 for 1811 points $\left(\chi^{2} / \mathrm{DOF}=1.07\right)$ in the case of CTEQ6M (abbreviated to C6M in the following) 1]. To gain a better feel of how these fits compare, we show in Table (the $3 \mathrm{rd}$ and 4 th columns) the overall $\chi^{2}$ values and, in parentheses, $\chi^{2}$ per data point, as well as for the applicable individual data sets. The total number of data points in this head-to-head comparison (not including the charm production data points) is 1925 . The new C6HQ fit reduces the overall $\chi^{2}$ by 29 out of $\sim 2000$ as compared to the C6M fit. The improvement of this generalized $\overline{M S}$ result over the zero-mass $\overline{M S}$ result is encouraging, since the generalized $\overline{M S}$ formalism represents a more accurate formulation of PQCD. However, a difference of $\chi^{2}$ of 29 is within the current estimated range of uncertainty

10 These data sets were not used in the CTEQ6M analysis because $F_{2}^{c}$ is not well-defined theoretically in the the zero-mass parton formalism. 


\begin{tabular}{|c|c|c|c|c|c|}
\hline 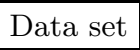 & $\mathrm{pts}$ & CTEQ6HQ & TEQ6M & $\mathrm{C} 6 \mathrm{M} \otimes \mathrm{GM}$ & 10 \\
\hline $\mathrm{dn}$ & 339 & $370(1.0$ & $370(1$. & $70(1.11)$ & $373(1.1$ \\
\hline & 2 & 269( & 9 & $274(1.07)$ & 1 \\
\hline & 1 & 94( & 102( & $258(2.84)$ & 387 \\
\hline & 12 & 124 & 130( & $135(1.11)$ & $123(0.9$ \\
\hline & 1 & 103( & 111 & $119(0.84)$ & $104(0.8 \mathrm{C}$ \\
\hline & 2 & 266 & 261 & $474(2.11)$ & $364(1.5 !$ \\
\hline & 201 & 304 & 299 & 273 & $366(1.8$ \\
\hline & 123 & 112( & 111 & $111(0.90)$ & $114 \quad 0.92$ \\
\hline & 69 & 0) & 120 & $116(1.82)$ & $107(1.5$ \\
\hline & 86 & 1 & 3 & 40) & $36(0.42$ \\
\hline & 119 & $102(\mathrm{C}$ & $103(0.86)$ & $101(0.86)$ & $102(0.86$ \\
\hline & 11 & & & $9(0.83)$ & $9(0.78$ \\
\hline & 15 & 5( & 3) & 43) & $5(0.34)$ \\
\hline & 90 & $71(\mathrm{c}$ & $49(0.55)$ & $49(0.55)$ & $71(0.7 \mathrm{~s}$ \\
\hline Cdf_jet & 33 & $55(1.66)$ & 50( & $50(1.51)$ & $55(1.66)$ \\
\hline All & 925 & $008(1.04)$ & 037 (1.06) & $431(1.26)$ & 2496 \\
\hline
\end{tabular}

TABLE I: Comparison of the $\chi^{2}$ values of the general-mass CTEQ6HQ fit (3rd column) with the zero-mass CTEQ6M fit (4th column). Also included are comparisons with two "mis-matched" cases when GM and ZM parton distribution functions are convoluted with the other (wrong) hard-cross-section (5th and 6th columns). The first number of each entry is the $\chi^{2}$ value, the number in parenthesis is $\chi^{2}$ per number of points. Correlated systematic errors, if available, are included.

of PDF analysis. 1, 23, 24] Therefore, the significance of this difference is arguable. We also note that the improvement in $\chi^{2}$ is spread over most of the data sets: there is no smoking gun for the overall difference.

The last two columns of Table \ compare the above results with two possible uses of the PDFs that represent a mis-use of PQCD in principle, but occur frequently in the literature in practice, perhaps out of necessity. These involve using PDFs obtained in the general-mass scheme convoluted with hard-scattering cross-sections (Wilson coefficients) defined in the zero-mass scheme, and vice versa. ${ }^{11}$ For the same data sets, these mis-matched schemes result in a difference of 420 490 in the overall $\chi^{2}$. These are quite large differences relative to the tolerances discussed in Refs. [1, 25], and results in clear discrepancies with some of the precision DIS data sets, as can be seen in Table The lesson is clear: for quantitative applications, it is imperative to maintain consistency between the PDFs and the hard-scattering cross-sections.

\section{COMPARISON WITH DATA}

In Ref. 1], we presented an extensive comparison between the CTEQ6 results and the data sets used in the global analysis, including new ways to explicitly account for the correlated experimental systematic errors. Since the new $\mathrm{C} 6 \mathrm{HQ}$ fit is generally similar to the C6M fit, we shall not duplicate the same comparisons for those quantities where the differences are minimal. In subsection IVA we compare with the DIS charm production data, which were not used in the previous CTEQ6 analysis. In subsection IVB we discuss some implications of the neutrino data.

${ }^{11}$ For instance, the MRST distributions [25] are obtained in the General-Mass formalism (using the Robert-Thorne implementation [16]); they are often used in applications convolving with readily available Zero-Mass hard-scattering cross-sections. 


\section{A. DIS Charm Production Data from HERA}

Because the $\mathrm{H} 1$ and ZEUS $F_{2}^{c}$ structure function are sensitive to the charm and gluon distributions, these will play an important role in extracting the C6HQ PDFs. In Fig. 1 we display comparisons of the charm production data from H1 20] and ZEUS [26] with the C6HQ theory value. For simplicity, we combine all the data points from each experiment in a single plot, and we scale the plot by the theoretical calculation. On the x-axis, the data points are ordered using $x$ and $Q$ as the primary and secondary sorting variables, respectively. The error bars represent the statistical and uncorrelated systematic errors added in quadrature. We see that the fits are good with $\chi^{2}$ per data point of $0.881(7 / 8)$ for H1, and $0.98(18 / 18)$ for ZEUS, respectively.
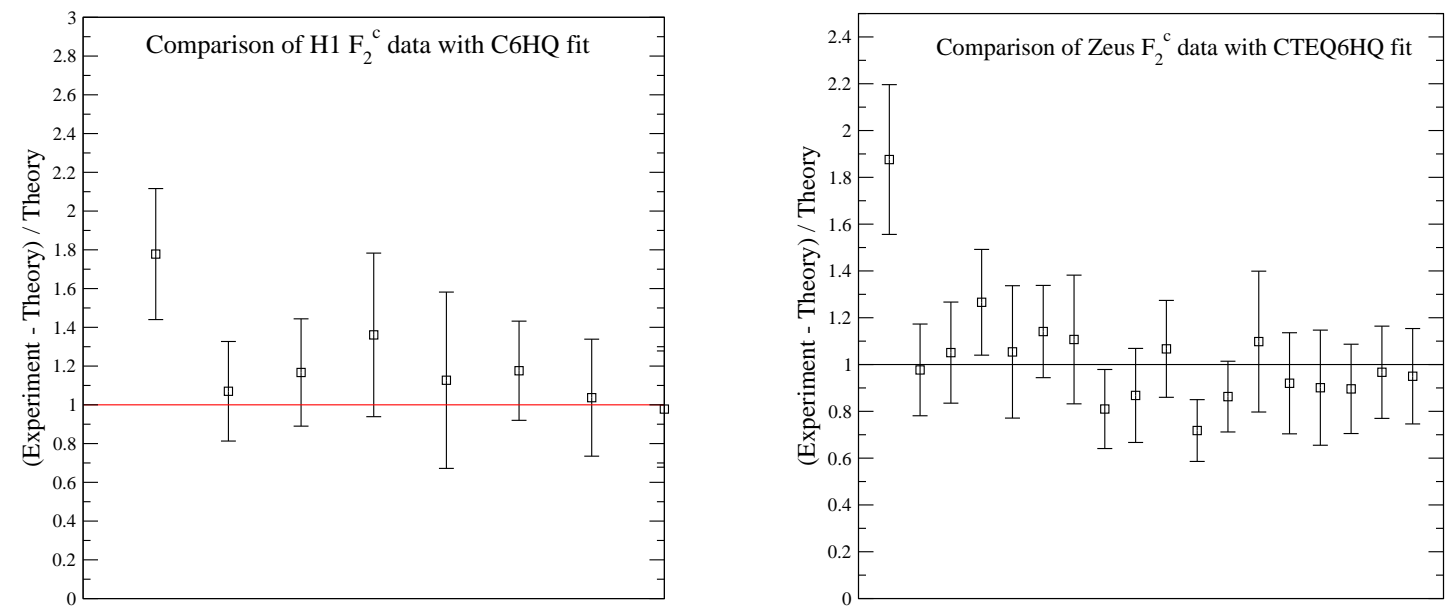

FIG. 1: Comparisons of the charm production structure function, $F_{2}^{c}$, with the data from H1 (Fig. [1. 20] and ZEUS (Fig. 1.b). 26] The $y$-axis is (Experiment-Theory)/Theory; along the x-axis, the data points are ordered using $x$ and $Q$ as the primary and secondary sorting variables respectively. The error bars represent the statistical and uncorrelated systematic errors added in quadrature.

\section{B. Neutrino Data in Global Parton Analyses}

The NuTeV measurement of the weak mixing angle $\sin ^{2} \theta_{\mathrm{W}}$ (Ref. 27] ) has recently focused considerable attention on the neutrino induced DIS process. 28] In this measurement, the weak mixing angle $\sin ^{2} \theta_{\mathrm{W}}$ is extracted from the ratio of neutral current (NC) to charged current (CC) $\nu$ and $\bar{\nu}$ cross-sections. Even before this $\sin ^{2} \theta_{\mathrm{W}}$ measurement, there have been other long-standing unresolved issues in comparing CC structure functions $F_{2}^{C C}$ (measured in $\nu$ and $\bar{\nu}$ scattering) with $\mathrm{NC}$ structure functions $F_{2}^{N C}$ (measured in $e^{ \pm}$and $\mu^{ \pm}$scattering) at modestly low- $x\left(\sim 10^{-2}\right)$. One manifestation of this is that the recently measured structure function $\Delta x F_{3}^{\nu / \bar{\nu} N}$ is not compatible with the QCD predictions. 29, 30] As this structure function is particularly sensitive to the heavy quark components, $\Delta x F_{3}^{\nu / \bar{\nu} N} \simeq s(x)-c(x)$, it is certainly important to properly treat the heavy quark mass. While the deviations of $\Delta x F_{3}^{\nu / \bar{\nu} N}$ are at a non-dramatic $\sim 1 \sigma$ level, the pattern has been systematic and persistent.

Therefore, we re-examine the influence of the neutrino structure functions in the current global analysis keeping in mind the following issues. First, the high statistics neutrino structure function data of CCFR used 
here are from the newer "physics model independent" data analysis 31], rather than the earlier ones which contained model-dependent corrections. 32] Secondly, meaningful (i.e., quantitative) comparison of NC and CC structure functions (which represent different hard-scattering processes) can be made only within a common underlying theoretical framework with an accuracy comparable to the experimental uncertainties. ${ }^{12}$ The proper treatment of charm mass effects, such as those described in the current study, are an important part of that theoretical formalism, cf. 33 .

First, we ask whether the neutrino structure function, $F_{2}^{\nu / \bar{\nu} N}$, obtained by the newer modelindependent analysis is consistent with the muon-induced $\mathrm{NC}$ fixed-target structure functions, $F_{2}^{\mu^{ \pm} N}$, and the HERA collider structure functions, $F_{2}^{e^{ \pm}} N$, in a consistent NLO analysis. ${ }^{13}$ Fig. 2 2 shows the comparison between the data with the CTEQ6HQ fit (using $m_{c}=1.3 \mathrm{GeV}$ ). We see that, even if this data set is
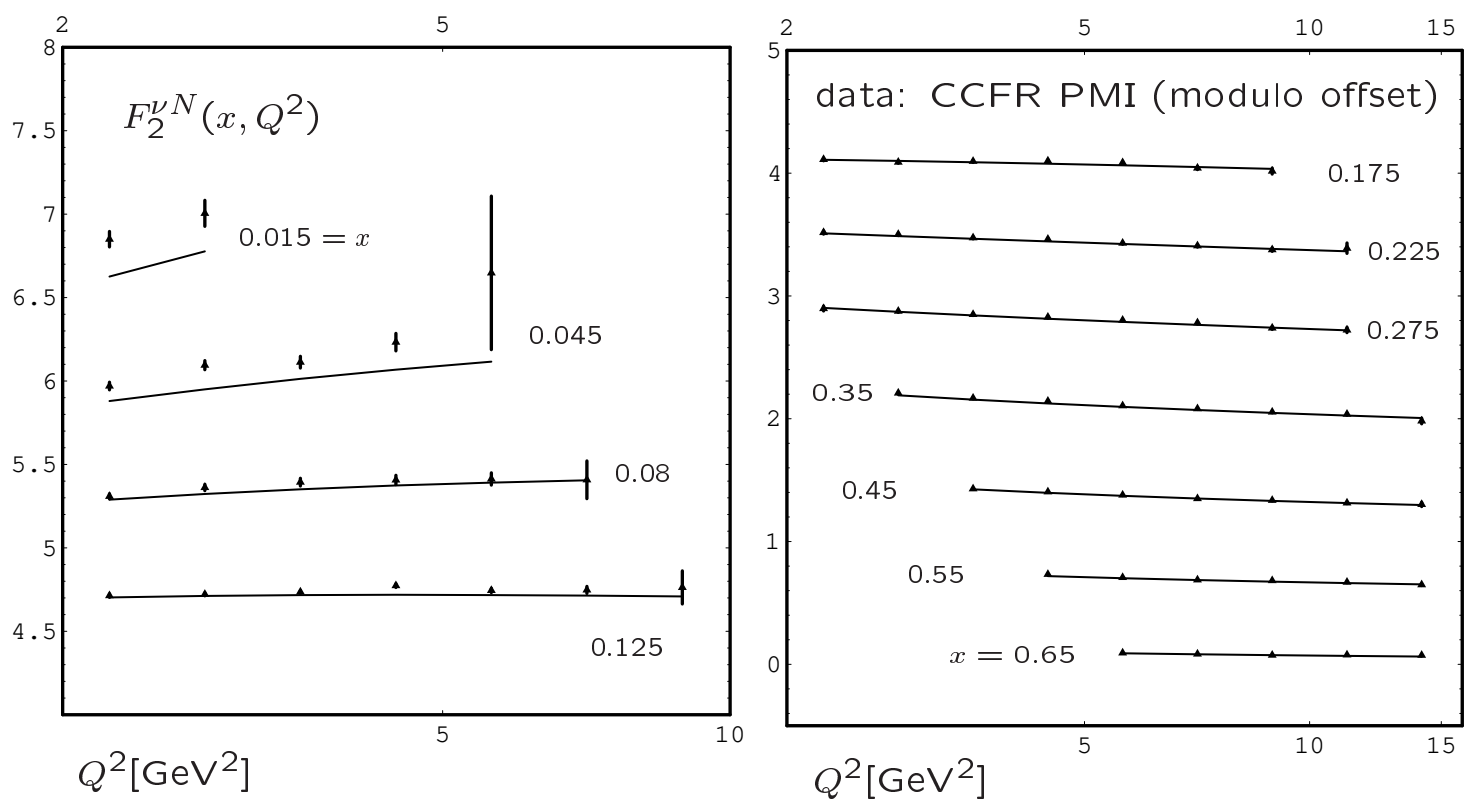

FIG. 2: CCFR $F_{2}^{\nu N}$ structure function (from the physics model independent analysis, Ref. 31]) compared to CTEQ6HQ fit: (a) low $x$ bins (left panel) show a systematic disagreement; (b) medium to high $x$ bins (right panel) show good agreement.

included in the global fit (cf., Table \), the measured values in the low- $x$ bins (left figure) consistently lie above the theory curves. The systematic deviation of data points from the global fit is reflected in the relatively large $\chi^{2}$ 's associated with this data set, as seen in Table 1 The fit is dominated by the much more extensive NC data (from the BCDMS, NMC, H1, and ZEUS experiments), hence one can regard the theory curves as representing the NC data properly "corrected" for NLO QCD effects. The pattern of deviation of the neutrino data from the average NC predictions at low- $x$ is similar to earlier comparisons. In other

12 For example, direct comparisons of the ratio $F_{2}^{\nu} / F_{2}^{\mu}$ to theory (suggested by the "5/18-rule") are meaningful only at the LO parton model level, which is not appropriate for the precision data from current experiments.

13 Ref. [31] suggests these data are consistent by examining the ratio of the structure functions. However, as explained in the text, it is not appropriate to compare the ratio of two different structure functions with theory in QCD beyond LO; a complete analysis involving a NLO fit is required. 
words, the discrepancy between CCFR $F_{2}^{\nu}$ and the other $\mathrm{NC} F_{2}^{e, \mu}$ measurements at small $x$ persists in the context of contemporary global QCD analyses, even when charm mass effects are properly treated as in the present analysis. Other possible theoretical sources for this difference (in addition to the NLO contributions included in this comparison) have been discussed in Refs. 28, 29, 34], and in the literature quoted therein. On the experimental side, preliminary NuTeV measurements [35] seem to yield improved agreements with the NLO QCD predictions at low- $x$. It remains to be seen whether the final $\mathrm{NuTeV}$ results will resolve this problem.

In our analysis, the strangeness distribution is constrained to be proportional to $[\bar{u}(x)+\bar{d}(x)]$; and its normalization at the scale $Q_{0}$ is constrained to be

$$
\kappa=\frac{\int d x x[s(x)+\bar{s}(x)]}{\int d x x[\bar{u}(x)+\bar{d}(x)]} \simeq \frac{1}{2},
$$

as in most current global analysis. In the future, a NLO analysis of the CC charm production data $(\nu s \rightarrow$

$\left.c \mu \rightarrow \mu^{ \pm} \mu^{\mp} X\right)$ from CCFR and NuTeV [36] has the potential to determine $\{s(x), \bar{s}(x)\}$ with much more precision. Then, a re-assessment of the apparent discrepancy between the measured $\Delta x F_{3}^{\nu / \bar{\nu} N}$ and the lowlying QCD predictions [29] will be warranted. An accurate determination of the strangeness of the proton, as well as the strangeness asymmetry $[s(x)-\bar{s}(x)]$ will have important implications for these measurements, including the NuTeV anomaly, cf., Refs. [27, 28].

Clearly, the interpretation of the neutrino DIS data requires a detailed NLO analysis. Global QCD analyses of parton distributions, such as the one performed in this paper, are essential to unraveling the underlying physics. Progress on both the experimental and the theoretical fronts are needed; this advancement is ongoing.

\section{COMPARISON WITH RELATED PDFS: CTEQ6HQ, CTEQ6M, CTEQ5HQ}

The CTEQ6HQ and CTEQ6M fits provide comparable descriptions of the global QCD data in two different schemes. Some of the differences in the PDFs arise purely from the choice of scheme. We are particularly interested in the differences for the charm distribution, and the closely correlated gluon distribution, due to the improved treatment of heavy quark effects in the generalized $\overline{M S}$ scheme. It is also interesting to compare the differences between the earlier (previous generation) CTEQ5HQ (C5HQ) distributions with the new CTEQ6HQ distributions; differences between these PDFs are attributable both to new data, and to minor differences in the way the theoretical inputs are implemented. We combine these comparisons in the figures presented below.

\section{A. Light partons at the input scale}

Figs. 33,b show the $u$-quark and $d$-quark distributions from C5HQ, C6M and C6HQ at the initial $Q$-scale of $1.3 \mathrm{GeV}$ (the charm mass). In order to exhibit the behavior of the PDFs at both large and small $x$ values, the $\mathrm{x}$-axis is scaled linearly in $\sqrt{x}$. To give some additional physical insight to these plots, the $\mathrm{y}$-axis is chosen to be $x^{1.5} f(x, Q)$, so that the area under each curve represents the momentum fraction carried by that distribution. This chosen scaling then focuses on the valence peak, and suppresses the small- $x$ sea excitations. We see that the valence $u$-quark and $d$-quark distributions are well constrained by the precision DIS data; the scheme dependence is not pronounced.

Figs. 4a,b show the same PDFs, except the axes are scaled differently in order to highlight the sea quark distributions. The differences between the CTEQ5 and CTEQ6 generations of PDFs are a result of the improved low- $x$ data. When we compare the HQ and $\overline{M S}$ type PDFs of the CTEQ6 generation, we observe the general pattern that the HQ distributions overshoot the $\overline{M S}$ ones at low $x\left(\sim 10^{-3}\right)$, and that the distributions meet again at ultra-small $x\left(\lesssim 10^{-5}\right)$. We can qualitatively understand the larger sea quark 

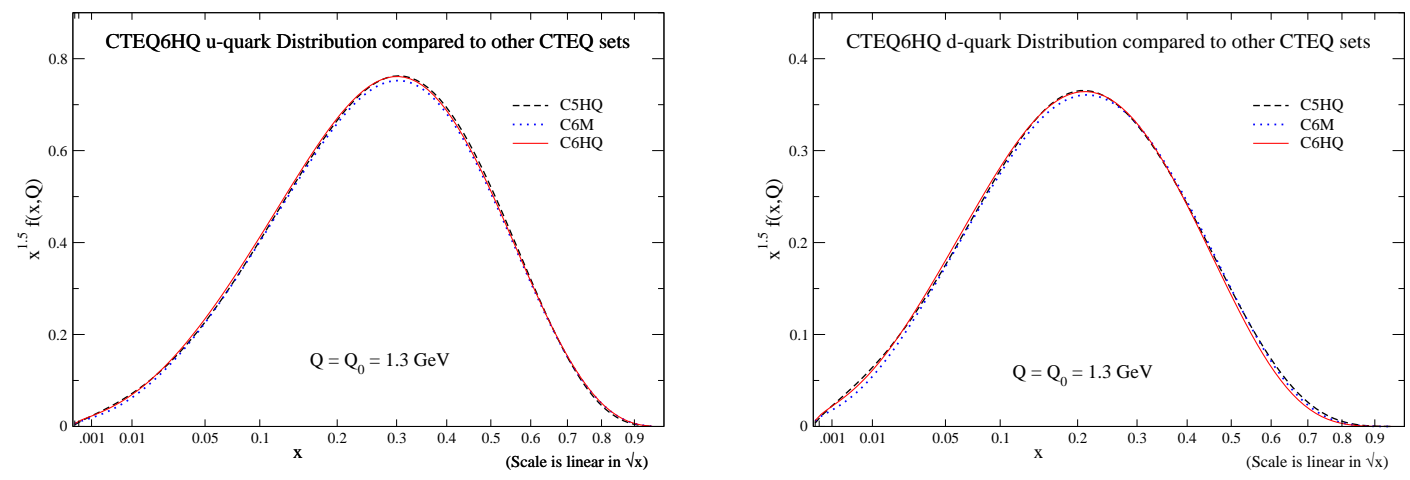

FIG. 3: Comparison of CTEQ5HQ, CTEQ6M, and CTEQ6HQ parton distributions at $Q=m_{c}=1.3 \mathrm{GeV}$ : (a) u-quark; and (b) d-quark. The axes are scaled to highlight the valence components of these distributions.
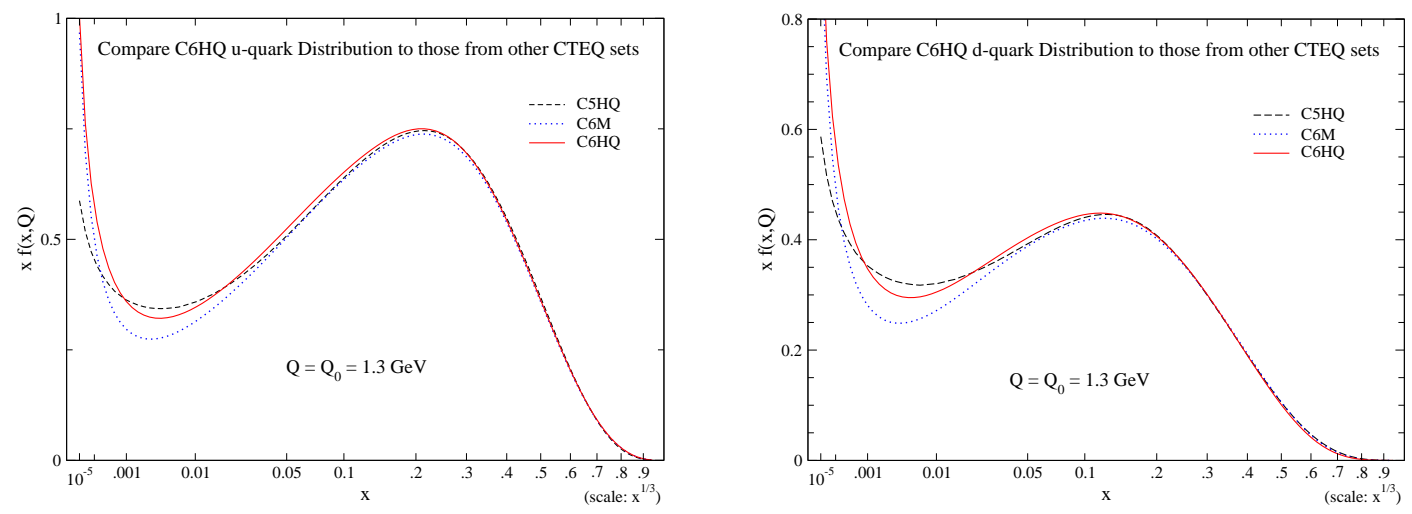

FIG. 4: Same as Fig. [3 except the axes are scaled to highlight the sea components of the PDFs: (a) u-quark; and (b) d-quark.

distributions for the HQ scheme since these distributions are compensating for the suppression of charm in this scheme; this suppression is absent in the zero-mass $\overline{M S}$ scheme. In the region of ultra-small $x$, there is no data constraining these distributions; we show this effect graphically in the next Section where we consider the PDF uncertainty band of the the $\mathrm{HQ}$ and $\overline{M S}$ distributions.

Fig. [5] shows the comparison of the gluon distribution at $Q_{0}$. Here the difference between $\mathrm{C} 5 \mathrm{HQ}$ and the CTEQ6 generation of gluon distributions is pronounced. As discussed in Ref. [1], the change in this least-well-determined parton distribution is due to the recent precision DIS data (most influential in the small $x$ region) in conjunction with the greatly improved inclusive jet data from the Tevatron (critical for the medium to large $x$ regions). The differences between $\mathrm{C} 6 \mathrm{HQ}$ and $\mathrm{C} 6 \mathrm{M}$ gluons at large $x$ are due to a combination of scheme-dependence, and the inherent uncertainty range of the current analysis. [1]

Fig. [6 shows the comparison of the strange distributions at the same $Q_{0}$. The noticeable difference between the C5HQ curve and the others, in this case, is largely the result of different theoretical inputs: 


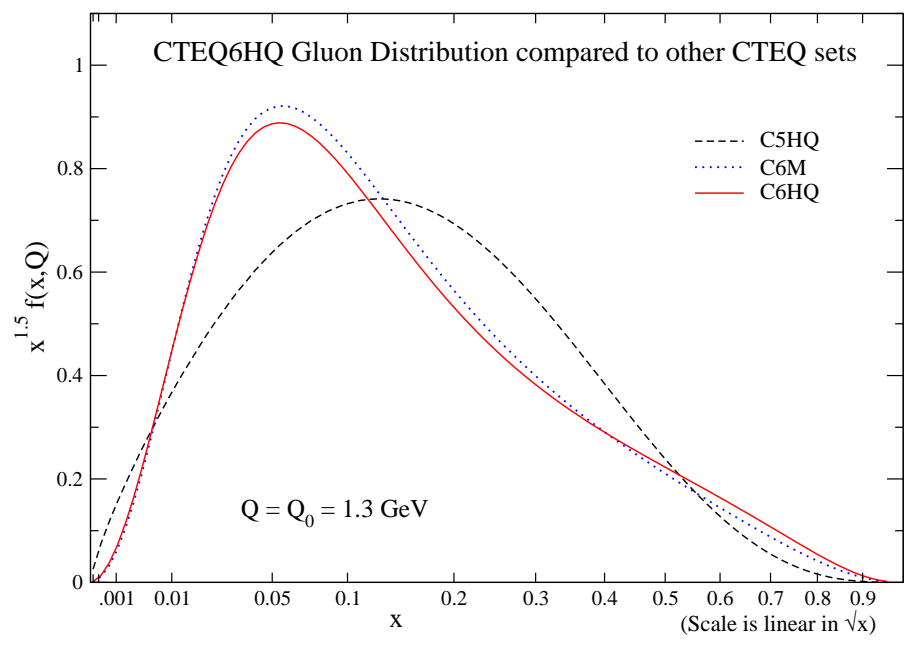

FIG. 5: Comparison of the gluon distributions at $Q_{0}=1.3 \mathrm{GeV}$.

namely, the $\kappa$ parameter (cf., Eq. [8). $\kappa$ determines the ratio of strange to non-strange sea quarks at the initial scale $Q_{0}$. This $\kappa$ factor, known only approximately, was chosen to be $1 / 2$ both in the CTEQ5 and CTEQ6 analyses, but for slightly different values of $Q_{0}-1.0 \mathrm{GeV}$ for C5HQ and $1.3 \mathrm{GeV}$ for CTEQ6 sets.

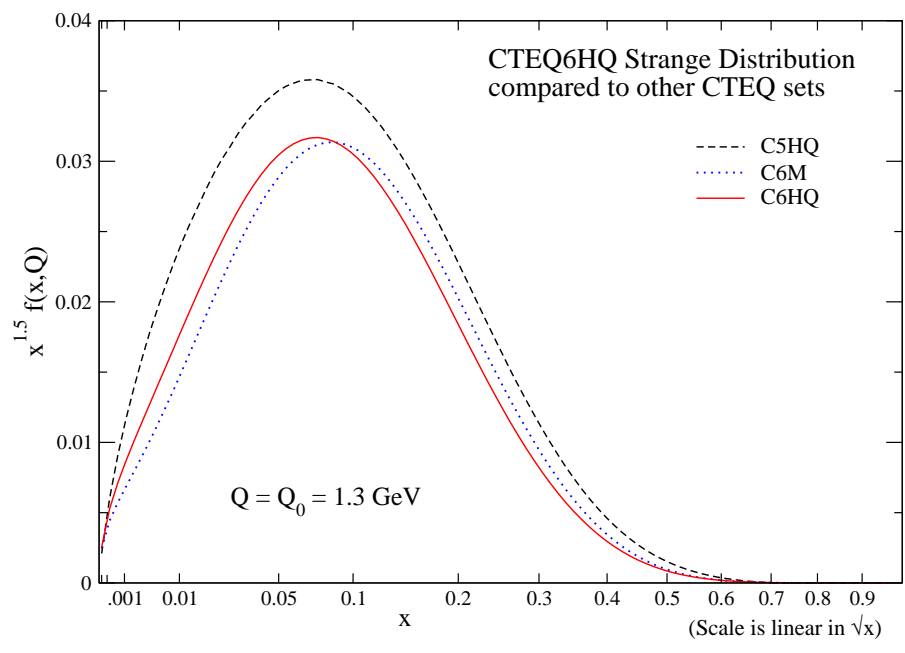

FIG. 6: Comparison of the strange distributions at $Q_{0}=1.3 \mathrm{GeV}$. 

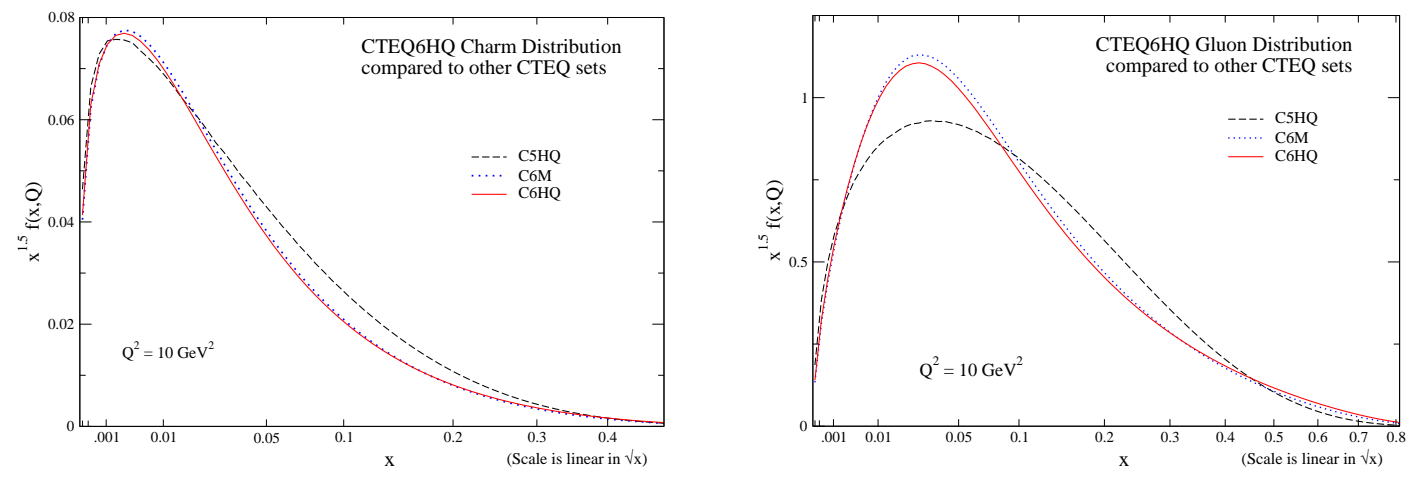

FIG. 7: Comparison of the charm and gluon distribution at $Q^{2}=10 \mathrm{GeV}^{2}$.

\section{B. Charm and gluon distributions at $Q^{2}=10 \mathrm{GeV}^{2}$}

To compare the differences of the charm distributions, we need to move some distance above the charm mass scale, since all current sets assume a zero charm distribution at $\mu=m_{c}$. Fig. [7 makes this comparison at $Q^{2}=10 \mathrm{GeV}^{2}$. We see a substantial difference between the C5HQ and the two CTEQ6 charm distributions. Unlike the case of the strange quark, this difference is physical; it is mainly a reflection of the difference in the gluon distributions (shown already for a lower $Q$ value in Fig. 5) because charm is radiatively generated from the gluon. To confirm this hypothesis, Fig. $7 \mathrm{~b}$ shows the gluon distributions at the same $Q^{2}=10 \mathrm{GeV}^{2}$; the similarity is clear.

\section{C. $Q$-dependence of heavy quark mass effects}

In this subsection, we compare the $Q$ dependence of the C6M and $\mathrm{C} 6 \mathrm{HQ}$ parton distributions at fixed values of $x$ in order to see how the differences between PDFs in the zero-mass and general-mass schemes vary with increasing $\mu$-scale. We expect these differences (relative to the general PDF uncertainties) will decrease with increasing $Q$. The purpose of this study is two-fold. First, we check the self-consistency of the analysis, which is based on the expectation that power suppressed mass terms vanish asymptotically as $Q \rightarrow \infty$. Secondly, we determine the specific scales where the mass-effects become insignificant, in practice. The latter question is relevant for high-energy applications of PDFs derived from lower-energy data. ${ }^{14}$ It can also be seen as a general illustrative example demonstrating how the relative importance of power suppressed terms decrease in the high $Q^{2}$ region where the conventional zero-mass PQCD results becomes dominant.

To examine the effects due to heavy flavor masses in the theoretical formalism, we cannot directly compare physical quantities that are being fit - they are the same (within the errors of the global fit) by construction. On the other hand, parton distribution function of individual flavors are not a good gauge either, because they are not physical - they are scheme dependent. Thus, for this comparison, we compromise and examine the quark singlet combination $\left(\Sigma \equiv \sum_{q}(q+\bar{q})\right)$ and the gluon distribution.

${ }^{14}$ It also serves to validate the self-consistency of analyses where threshold effects have been neglected (such as for collider processes). 


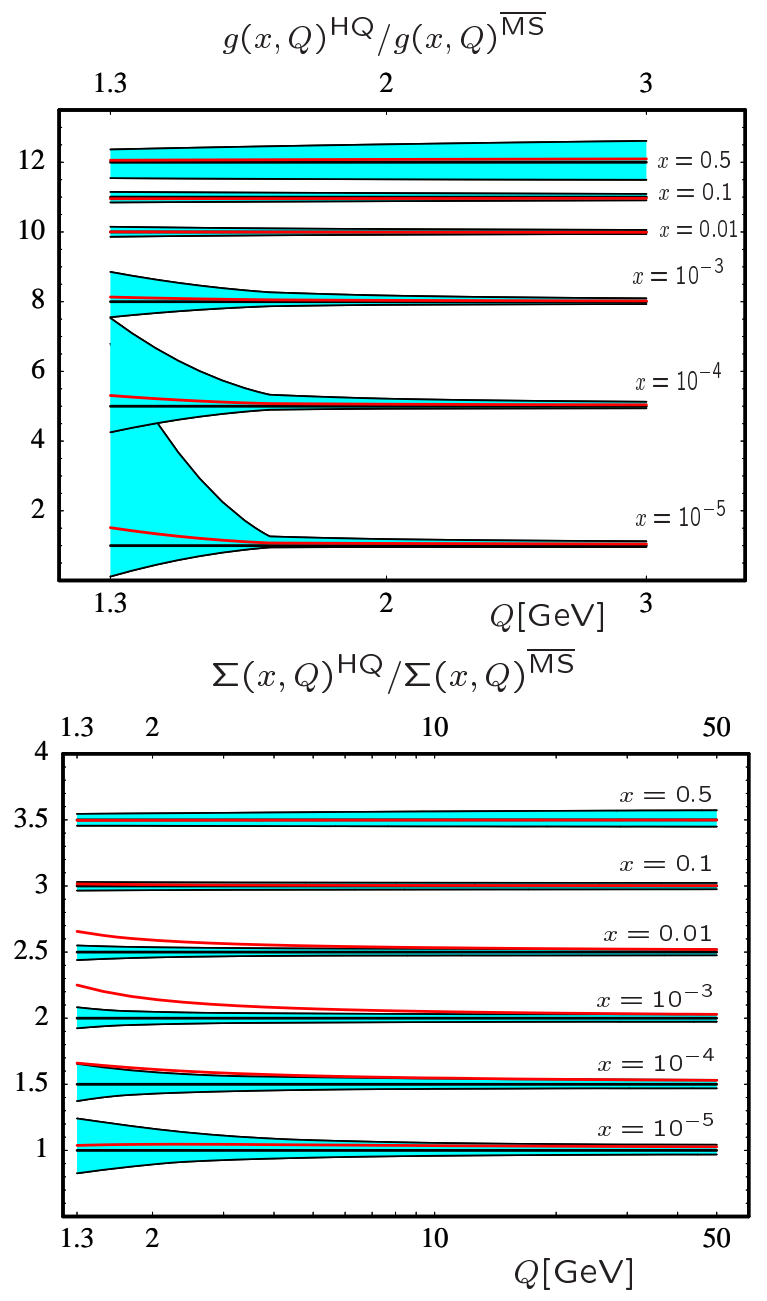

FIG. 8: Ratio of GM (C6HQ) to ZM (C6M) parton distributions as a function of $Q$ [GeV] along with the uncertainty band of the latter. The offset on the y-axis is arbitrary; i.e. the horizontal lines all correspond to $\Sigma(x, Q)^{\mathrm{HQ}} / \Sigma(x, Q)^{\overline{\mathrm{MS}}}=1$. Plotted are ratios for $x=\left\{10^{-5}, 10^{-4}, 10^{-3}, 10^{-2}, 0.1,0.5\right\}$ where $x$ is increasing from the lowest to the uppermost curve. We display: (a) the gluon; and (b) the singlet quark $\Sigma \equiv \sum_{q}(q+\bar{q})$

Figs. [ 8 a,b show, as solid lines, the $\mathrm{C} 6 \mathrm{HQ}$ gluon and quark singlet distributions normalized to that of C6M. The shaded bands represent the ranges of uncertainty of the relevant quantities due to experimental sources, as estimated in the CTEQ6 global analysis. 1] We observe the following pattern: for most of the parameter space, the deviation of the $\mathrm{C} 6 \mathrm{HQ}$ from $\mathrm{C} 6 \mathrm{M}$ falls within the estimated uncertainty range. In particular, for the gluon distribution this is true for all values of $x$. For the quark distribution, the HQ fit overshoots the zero-mass fit significantly (i.e., beyond the uncertainty band) at moderately low $x$ $\sim\left[10^{-2}, 10^{-3}\right]$. At very low $x \sim 10^{-5}$, the differences fall within the uncertainty bands again (primarily due to the increasing uncertainty). Although the HQ quark PDFs at $x \sim\left[10^{-2}, 10^{-3}\right]$ eventually evolve into the error band of the zero-mass fit as $Q$ increases, one may wonder why the two fits do not merge faster. The GM-VFNS and ZM-VFNS differ by the manner in which they organize terms of order $\mathcal{O}\left(\mathrm{m}^{2} / Q^{2}\right)$. Thus, the Wilson coefficients in the two schemes will be comparable when $\left(m^{2} / Q^{2}\right)<<1$. The boundary conditions 
of the PDFs at $Q_{0}$ will also differ between the two schemes by terms of order $\mathcal{O}\left(\mathrm{m}^{2} / Q^{2}\right)$; however, since the PDFs evolve logarithmically in the scaling variable, while the power corrections fall off more quickly, this can contribute to scheme differences at scales significantly above the scale $m$. This effect is evident in the case of the singlet quark PDF at moderately low $x$ values. Thus, the observed behavior is quite natural.

\section{CONCLUDING REMARKS}

The CTEQ6HQ PDFs presented here complement the previously published CTEQ6 sets by providing distributions which can be used in the generalized MS-bar scheme with non-zero mass partons. This analysis includes the complete set of NLO processes including the real and virtual quark-initiated terms. Additionally, the $\operatorname{ACOT}(\chi)$ scheme is used to introduce a generalized scaling variable which provides numerically stable results for the entire energy range - from heavy quark thresholds to the high energy limit.

While the zero-mass parton scheme is sufficient for many purposes, the fully massive scheme can be important when physical quantities are sufficiently sensitive to heavy quark contributions. This is evident when comparing the CTEQ6HQ and CTEQ6M fits to the mis-matched sets (Table 1) where the precise DIS data from HERA highlights the discrepancies.

The CTEQ6HQ fits also provide the basis for a series of further studies involving more quantitative analysis of strange, charm, and bottom quark distributions inside the nucleon. For example, the CTEQ6HQ PDFs are necessary for a consistent analysis of resummed differential distributions for heavy quark production such as in Ref. 37]. Using the full range of data from both the charged and neutral current processes, these

distributions can reduce the uncertainties in the calculations; hence, they have significant implications for charm and bottom production, and can help resolve questions about intrinsic heavy quark constituents inside the proton, the $\Delta x F_{3}$ structure function, and the extraction of $\sin \theta_{W}$.

\section{Acknowledgment}

We thank our colleagues J. Huston, P. Nadolsky, J. Pumplin, and D. Stump, for fruitful collaboration on the CTEQ6 project that forms the foundation of this study. F.O. acknowledges the hospitality of MSU and BNL where a portion of this work was performed. S.K. is grateful to RIKEN, Brookhaven National Laboratory and the U.S. Department of Energy (contract No. DE-AC02-98CH10886) for providing the facilities essential for the completion of this work. This research was supported by the National Science Foundation (grant No. 0100677), and by the Lightner-Sams Foundation.

[1] J. Pumplin, D. R. Stump, J. Huston, H. L. Lai, P. Nadolsky and W. K. Tung, JHEP 0207, 012 (2002) arXiv:hep-ph/0201195.

[2] J. C. Collins and W. Tung, Nucl. Phys. B278, 934 (1986).

[3] F. I. Olness and W. Tung, Nucl. Phys. B308, 813 (1988); M. A. Aivazis, F. I. Olness and W. Tung, Phys. Rev. Lett. 65, 2339 (1990).

[4] M. A. Aivazis, J. C. Collins, F. I. Olness and W. K. Tung, Phys. Rev. D 50, 3102 (1994) arXiv:hep-ph/9312319.

[5] J. C. Collins, Phys. Rev. D 58, 094002 (1998) arXiv:hep-ph/9806259.

[6] J. Amundson, C. Schmidt, W. K. Tung and X. Wang, JHEP 0010, 031 (2000) arXiv:hep-ph/0005221.

[7] E. Laenen, S. Riemersma, J. Smith and W.L. van Neerven, Nucl. Phys. B392 (1993) 162.

[8] M. Glück, E. Reya and M. Stratmann, Nucl. Phys. B422, 37 (1994).

[9] E. Witten, Nucl. Phys. B 104, 445 (1976).

[10] J. C. Collins, F. Wilczek and A. Zee, Phys. Rev. D 18, 242 (1978).

[11] M. Buza et. al., Eur. Phys. J. C1, 301 (1998) ; and M. Buza et. al., Phys. Lett. B411, 211 (1997). 
[12] A. Chuvakin et. al., Phys. Rev. D61, 096004 (2000).

[13] M. Cacciari et. al., JHEP 9805, 007 (1998). M. Cacciari, S. Frixione and P. Nason, JHEP 0103, 006 (2001) hep-ph/0102134.

[14] W. K. Tung, S. Kretzer and C. Schmidt, J. Phys. G 28, 983 (2002) arXiv:hep-ph/0110247.

[15] S. Kretzer, C. Schmidt, W. K. Tung et al., paper under preparation.

[16] R. S. Thorne and R. G. Roberts, Phys. Rev. D57, 6871 (1998); and Phys. Lett. B 421, 303 (1998) hep-ph/9711223.

[17] S. Kretzer and I. Schienbein, Phys. Rev. D 58, 094035 (1998) arXiv:hep-ph/9805233.

[18] M. Kramer, F. I. Olness and D. E. Soper, Phys. Rev. D 62, 096007 (2000) arXiv:hep-ph/0003035.

[19] C. Adloff et al. [H1 Collaboration], Eur. Phys. J. C 13, 609 (2000) arXiv:hep-ex/9908059.

[20] C. Adloff et al. [H1 Collaboration], Phys. Lett. B 528, 199 (2002) arXiv:hep-ex/0108039].

[21] J. Breitweg et al. [ZEUS Collaboration], Eur. Phys. J. C 12, 35 (2000) arXiv:hep-ex/9908012.

[22] S. J. Brodsky, P. Hoyer, C. Peterson and N. Sakai, Phys. Lett. B93, 451 (1980);

S. J. Brodsky, C. Peterson and N. Sakai, Phys. Rev. D23, 2745 (1981); B. W. Harris, J. Smith, R. Vogt, Nucl. Phys. B461, 181 (1996);

J. F. Gunion and R. Vogt, UCD-97-14, LBNL-40399, hep-ph 9706252.

[23] J. Pumplin, D. Stump, R. Brock, D. Casey, J. Huston, J. Kalk, H.L. Lai, W.K. Tung; Phys. Rev. D65: 014013 (2002).

[24] D. Stump, J. Pumplin, R. Brock, D. Casey, J. Huston, J. Kalk, H.L. Lai, W.K. Tung; Phys. Rev. D65: 014012 (2002).

[25] A.D. Martin, R.G. Roberts, W.J. Stirling, R.S. Thorne, Eur.Phys.J. C23 73 (2002); and ibid C28 455 (2003).

[26] ZEUS Collab. (J. Breitweg et al.), Eur. Phys. J. C12: 35 (2000).

[27] NuTeV Collaboration, G.P. Zeller et al., Phys. Rev.Lett. 88, 091802, 2002.

[28] See e.g.:

S. Davidson, S. Forte, P. Gambino, N. Rius, A. Strumia, JHEP 0202, 037, 2002.

A full list of references with possible interpretations of [27] is beyond the scope of the present publication.

[29] S. Kretzer, F.I. Olness, R.J. Scalise, R.S. Thorne, U.-K. Yang, Phys. Rev. D64, 033003 (2001); and references therein.

[30] P. Vilain et al. [CHARM II Collaboration], Eur. Phys. J. C11, 19 (1999); A. O. Bazarko et al. [CCFR Collaboration], Z. Phys. C65, 189 (1995); A. O. Bazarko, Ph.D. Thesis. NEVIS-1504; S.A. Rabinowitz et al. [CCFR Collaboration], Phys. Rev. Lett. 70, 134 (1993); H. Abramowicz et al. [CDHSW Collaboration], C. Phys. C15, 19 (1982); P. Astier et al. [NOMAD Collaboration], Phys. Lett. B486 (1900) 35; M. Goncharov et al. [NuTeV Collaboration], Phys. Rev. D64, 112006 (2001).

[31] U.K. Yang et al. [CCFR/NuTeV Collab.], Phys. Rev. Lett. 86, 2742 (2001).

[32] W. G. Seligman et al., CCFR Collab., Phys. Rev. Lett. 79, 1213 (1997);

W. G. Seligman, Ph.D. Thesis, Columbia University, Nevis-292 (1997).

[33] M.A.G. Aivazis, F.I. Olness, W.-K. Tung, Phys. Rev. Lett. 65: 2339 (1990); M. Glück, S. Kretzer and E. Reya, Phys. Lett. B380, 171 (1996); B405, 391 (1996) (E); V. Barone, M. Genovese, N. Nikolaev, E. Predazzi and B. Zakharov, Phys. Lett. B328, 143 (1994); V. Barone, U. D'Alesio and M. Genovese, Phys. Lett. B357, 435 (1995).

[34] As a point of entry, see e.g.:

A.L. Kataev, La Thuile 2001, Results and perspectives in particle physics, 205-221, hep-ph/0107247 J.T. Londergan, A.W. Thomas, hep-ph/0301147 S. Kumano, Phys. Rev. D66, 111301 (2002); S. Kovalenko, I. Schmidt, J.-J. Yang, Phys. Lett. B546, 68 (2002).

[35] R. Bernstein [NuTeV Collab.], presentation at DIS2002, Cracow, 30 April - 4 May 2002.

[36] NuTeV Collaboration (M. Goncharov et al.), Phys. Rev. D64, 112006 (2001); S. Kretzer, D. Mason and F. Olness, Phys. Rev. D65, 074010 (2002).

[37] P. M. Nadolsky, N. Kidonakis, F. I. Olness and C. P. Yuan, arXiv:hep-ph/0210082 to appear in Phys. Rev. D. 